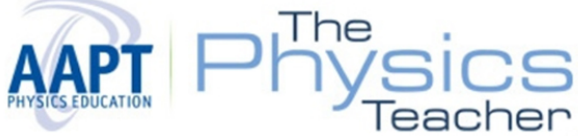

\section{Pharmacokinetics and RC Circuit Concepts}

Mieke De Cock and Paul Janssen

Citation: Phys. Teach. 51, 482 (2013); doi: 10.1119/1.4824943

View online: http://dx.doi.org/10.1119/1.4824943

View Table of Contents: http://tpt.aapt.org/resource/1/PHTEAH/v51/i8

Published by the American Association of Physics Teachers

\section{Related Articles}

FIGURING PHYSICS

Phys. Teach. 51, 503 (2013)

A glass of wine a day does not keep optics away! Reflection and refraction images in wine glasses

Phys. Teach. 51, 506 (2013)

On the Intensity Profile of Electric Lamps and Light Bulbs

Phys. Teach. 51, 491 (2013)

Smartphone-aided measurements of the speed of sound in different gaseous mixtures

Phys. Teach. 51, 508 (2013)

Archimedes in Action

Phys. Teach. 51, 498 (2013)

\section{Additional information on Phys. Teach.}

Journal Homepage: http://tpt.aapt.org/

Journal Information: http://tpt.aapt.org/about/about_the_journal

Top downloads: http://tpt.aapt.org/most_downloaded

Information for Authors: http://www.aapt.org/publications/tptauthors.cfm

\section{WebAssign.}

The PREFERRED Online Homework

\section{Solution for Physics}

Every textbook publisher agrees! Whichever physics text you're using, we have the proven online homework solution you need. WebAssign supports every major physics textbook from every major publisher.

webassign.net

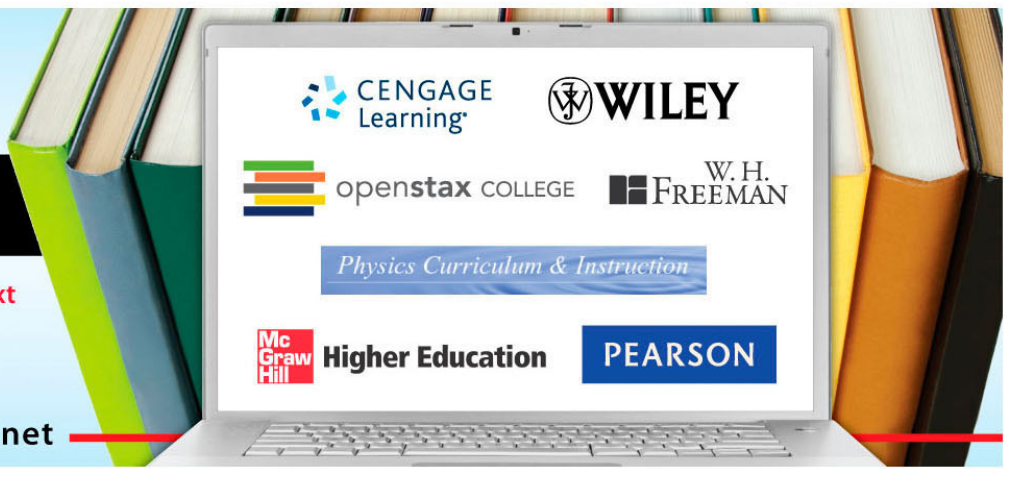




\section{Pharmacokinetics and $R C$ Circuit}

\section{Concepts}

\section{Mieke De Cock and Paul Janssen, KU Leuven, Belgium}

M ost introductory physics courses include a chapter on $R C$ circuits in which the differential equations for the charging and discharging of a capacitor are derived. A number of papers in this journal ${ }^{1-5}$ describe lab experiments dealing with the measurement of different parameters in such $R C$ circuits. In this contribution, we report on a lab experiment we developed for students majoring in pharmacy, using $R C$ circuits to simulate a pharmacokinetic process.

We see two benefits specific for this project. The first is to lift the $R C$ circuit above its usual electrostatic context by placing it amidst other phenomena, seemingly completely different but described by essentially the same differential equation and so exhibiting similar behavior. The use of a pharmaceutical model to illustrate this adds to the motivation of the students. The second pro is that the lab provides excellent training in the use of exponential functions. This is especially welcome in a curriculum where mathematics is not a first priority but in which exponential behavior will be met frequently and in many domains.

\section{A simple pharmacokinetic model}

Pharmacokinetics ${ }^{6}$ deals (among other items) with the speed of deactivation or elimination from the body of a medicine after its administration.

We present here a setup to mimic a very basic pharmacokinetic model. In this model, a patient is administered a fixed dose $d$ of a certain drug at regular intervals each of duration $t_{0}$, e. g., once a day. Administration takes place at the same moment every day. We model the body as one single compartment and we assume that all of the drug is absorbed and spread in the body instantaneously at the moment of administration. After intake, the drug will gradually be eliminated from the body. We do not attempt to explain the (sometimes complicated and maybe multiple) mechanisms of this. Instead, the most simple and most basic model in pharmacokinetics is followed, in which the drug elimination rate is proportional to the amount of drug present in the body. This model can be described by the differential equation

$$
\frac{d x}{d t}=-\alpha x,
$$

in which $x(t)$ is the amount of agent in the body at time $t$ and $\alpha$ is a constant. This process can be seen as follows (Fig. 1):

- Before the first administration, $x=0$;

- At time $t=t_{1}$, a first dose is administered, so $x_{1}=x\left(t_{1}\right)=d$;

- Between $t_{1}$ and $t_{2}$, the drug is eliminated (exponentially) and the amount decreases from $x_{1}$ to $x_{2}$;

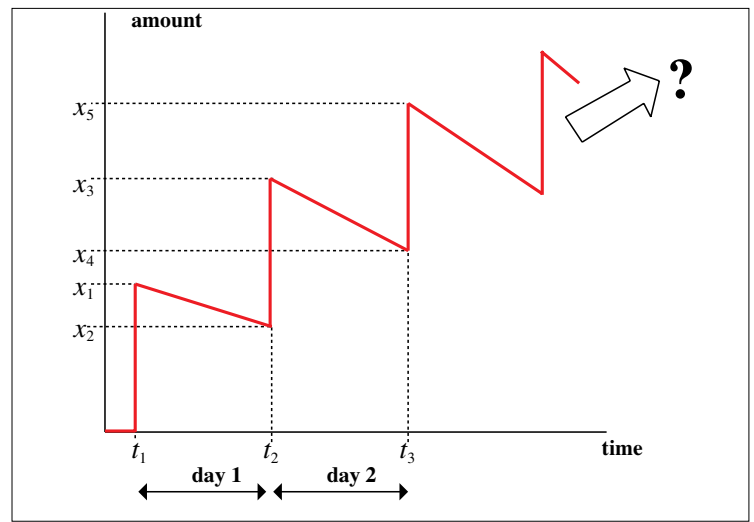

Fig. 1. Schematic time evolution of agent in the body.

- At time $t=t_{2}$, again a dose is administered, and so $x_{3}=$ $x\left(t_{2}+t_{\mathrm{L}}\right)=x_{2}+d$, where $t_{\mathrm{L}}$ is the time required for administration and spreading of the drug. We will assume $t_{\mathrm{L}}$ $\ll t_{0}$.

- Again, during the second day (between $t_{2}$ and $t_{3}$ ), $x$ decays exponentially, with the same time constant, to the value $x_{4}$;

- At time $t=t_{3}$, a new dose is injected, so $x_{5}=x_{4}+d$;

- This sequence can be repeated as long as is necessary.

We want to investigate the long time evolution of the amount of drug in the body, i.e., the amount after "many days."

\section{$R C$ circuit simulation}

To simulate this process, a setup as shown in Fig. 2 was developed. The amount of drug in the body is represented by the charge $q$ on a capacitor $C$. This amount can be calculated by measuring the potential difference $V$ across the capacitor and computing $q=C V$, with $C$ known.

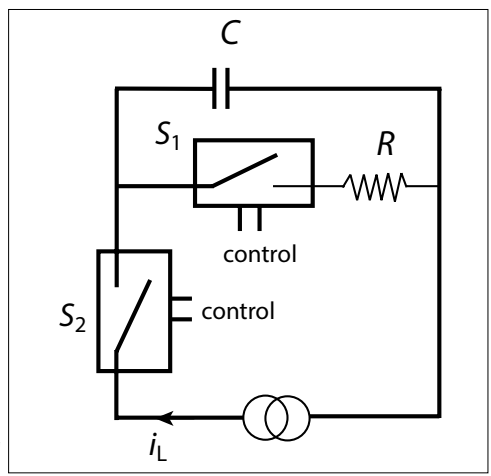

The capacitor is completely discharged before the measurement begins. The sequence to be used is then as follows:

1. Switch $S_{1}$ is opened and $S_{2}$ is closed. The system is kept in this state for a fixed time $t_{\mathrm{L}}$, the loading time. Note that, in order to have a constant current, independent of the charge already on the capacitor, we have to use a constant 
current source and not a constant voltage source. Writing $i_{\mathrm{L}}$ for the "loading" current, the charge delivered to the capacitor is $i_{\mathrm{L}} t_{\mathrm{L}}$, representing the dose $d$ in the theoretical model.

2. After loading, $S_{2}$ is opened (so no more charge can flow to the capacitor) and $S_{1}$ is closed. This allows the capacitor to discharge through the resistance $R$, with a time constant $\tau=R C$. The system is kept in this state for a time $t_{0}$. In order to mimic the "instantaneous" absorption of the drug, we should keep $t_{\mathrm{L}} \ll t_{0}$. If this is satisfied, $t_{0}$ can be taken as "one day."

To investigate the long time evolution, the phases of "drug administration" and "drug elimination" are consecutively repeated as "charging" and "discharging" the capacitor. The process is summarized in Fig. 3.

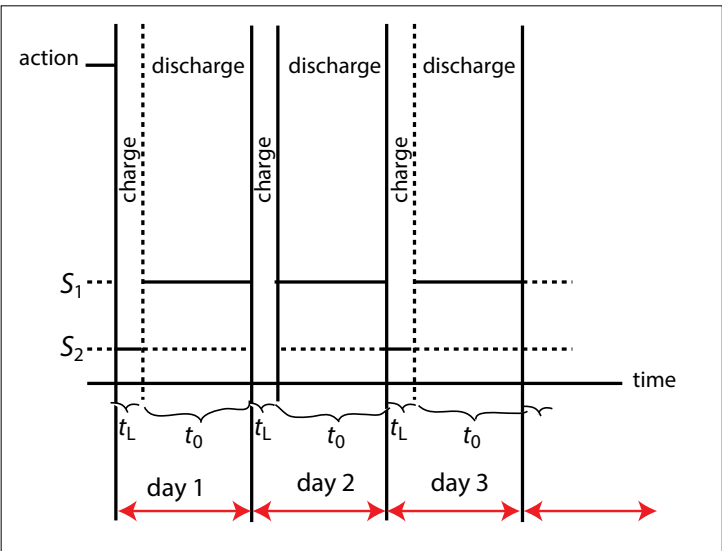

Fig. 3. Schematic representation of cycles of charging and discharging ( $t_{\mathrm{L}}$ is exaggerated for clarity).

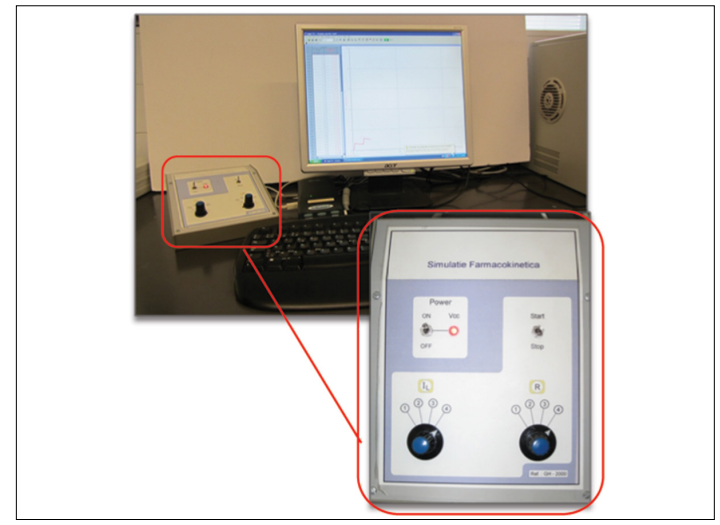

Fig. 4. Experimental setup.

Table I. Characteristics of experimental setup.

\begin{tabular}{|l|l|}
\hline $\mathrm{C}$ & $72 \mu \mathrm{F}(10 \%)$ \\
\hline$t_{\mathrm{L}}$ & $0.150+/-0.004 \mathrm{~s}$ \\
\hline$t_{0}$ & $10.0+/-0.3 \mathrm{~s}$ \\
\hline$i_{\mathrm{L}}$ & $172 / 344 / 520 / 700 \mu \mathrm{A}(5 \%)$ \\
\hline$R$ & $27 / 297 / 420 / 890 \mathrm{k} \Omega(2 \%)$ \\
\hline
\end{tabular}

\section{Experimental setup}

Figure 4 shows a picture of the actual setup and a closeup of the apparatus containing the $R C$-simulation part. The main characteristics are given in Table I. The control of $S_{1}$ and $S_{2}$ is done automatically inside the apparatus. The voltage across the capacitor is measured as a function of time using the Vernier LabPro interface. Analyzing the results is done using the Vernier Logger Pro software.

\section{Experiments and interpretation}

The value $C$ of the capacitor as well as $t_{0}$ and $t_{\mathrm{L}}$ are fixed in the apparatus. By changing $i_{\mathrm{L}}$ and $R$, both the dose and the rate at which the drug is eliminated can be changed and the long-term behavior can be studied in various situations.

For a first experiment, a time constant is chosen that is small compared to $t_{0}$. This mimics the situation of rapid drug

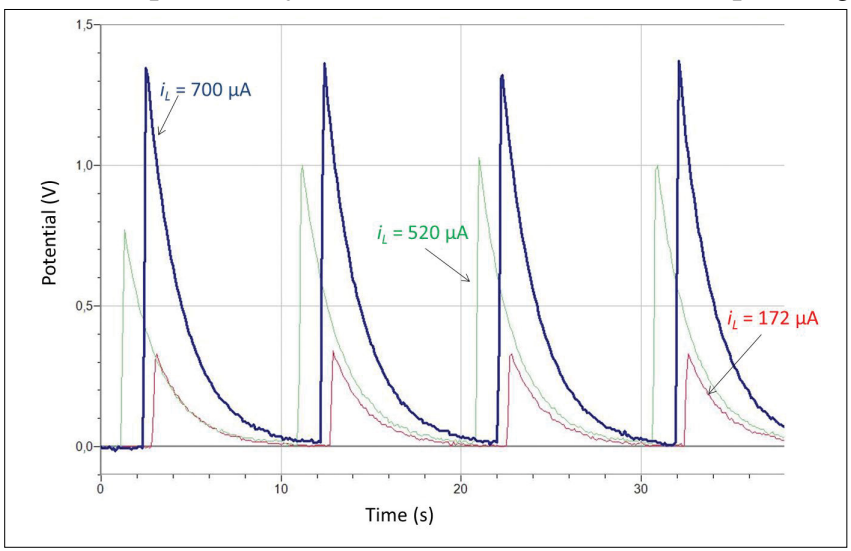

Fig. 5. Time evolution for a system with a small time constant and different doses.

elimination, where the amount of drug in the body decreases to the initial level before a next dose is administered. A superposition of three different records is shown in Fig. 5. For these three measurements, the same value of $R$ was used $(R=$ $27 \mathrm{k} \Omega$, corresponding to $\tau=1.94 \mathrm{~s}$ ). The dose was different in each case, corresponding to $i_{\mathrm{L}}=172 \mu \mathrm{A}, 520 \mu \mathrm{A}$, and $700 \mu \mathrm{A}$, respectively. The student is asked to give a qualitative explanation of the behavior seen (a simple task in this case). Furthermore, to become familiar with the equipment, the dose and the decay constant must be measured on the graph. This can be done by using the analysis tools in the software that allow one to determine minima and maxima and to perform a curve fitting on the exponential decay. Both quantities (i.e., $d$ and $\tau$ ) are to be compared with the values expected from the experimental parameters.

For the curve fitting, students have to cope with the fact that the time the computer assigns to events is not the same as the time that appears in the usual exponential decay formula. The PC and the control circuit for opening and closing $S_{1}$ and $\mathrm{S}_{2}$ are indeed not synchronized. This is clearly visible in Fig. 5. It can be used as a starting point for an interesting discussion with the inexperienced student on the number of independent parameters in the curve fitting. 
A different behavior is seen when the elimination is slow compared to the interval between two administrations. In that case, a significant amount of drug is still present in the body when the next dose is administered. This is simulated by a large time constant and the capacitor being not completely discharged when the next charging period starts.

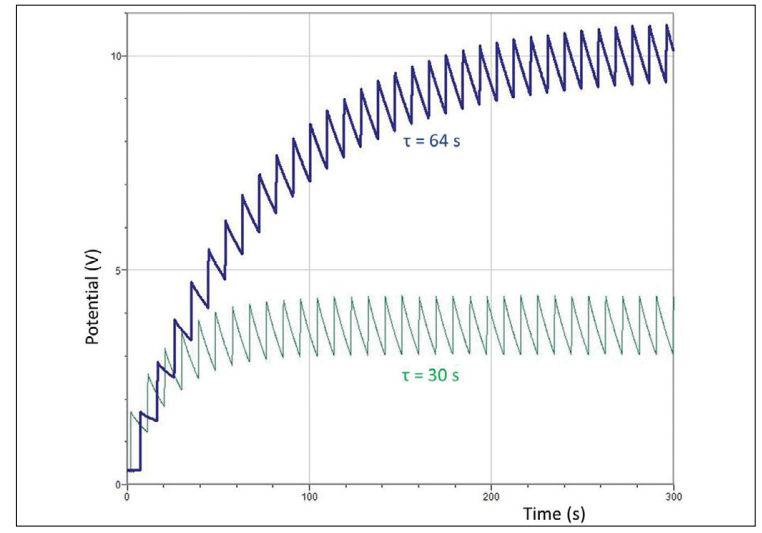

Fig. 6. Time evolution for system with a large time constant.

Figure 6 shows the voltage across the capacitor for two such situations with the same dose but a different time constant. Again both the dose and the time constant can be obtained experimentally and can be compared to the expected values. The overall behavior of the graph comes to many students as a surprise. Judging too hastily based on Fig. 1 makes them expect the charge to be ever increasing. Giving a qualitative explanation here is more difficult than before. The student must realize that the amount of charge lost by the capacitor in a fixed time interval (i.e., $t_{0}$ ) is proportional to the charge present at the beginning of that time interval. As the capacitor picks up more charge, the amount lost during "one day" is also increasing and eventually a steady state is established, when the amount of charge lost in one cycle equals the amount gained in the same cycle, i.e., when the loss equals the dose. This transition to a steady state is clearly visible in Fig. 6 . The student can check this by reading off the screen how the amount lost during a day varies over time and by comparing this to the dose.

In the steady state, it is interesting to obtain values for the minimum and maximum charge. One has $q_{\max }=q_{\min }+d$ (under steady state conditions!) and of course $q_{\min }=q_{\max } e^{-t_{0} / \tau}$
From these two:

$$
q_{\min }=d /\left(1-e^{-t_{0} / \tau}\right) \quad \text { and } \quad q_{\min }=d /\left(e^{t_{0} / \tau}-1\right) .
$$

This provides some clue about the average dose in the patient's body in the case of longtime drug intake. Again, this is to be worked out and verified on the graph in various situations.

\section{Conclusion}

This lab uses an $R C$ circuit to simulate a pharmacokinetic process and as such allows the transfer of a model describing a typical physical process to a process in a completely different field. For many non-science majors, it is exactly this aspect of mathematical modeling in physics that is important, much more than individual facts.

\section{References}

1. H. T. Wood, "The RC Circuit - A multipurpose laboratory experiment," Phys. Teach. 31, 372-373 (Sept. 1993).

2. J. G. King and A. P. French, "Using a multimeter to study an $R C$ circuit," Phys. Teach. 33, 188-189 (March 1995).

3. M. J. Moelter and R. S. Worland, "A child's flashlight and $R C$ circuit concepts," Phys. Teach. 35, 92-94 (Feb. 1997).

4. F. X. Hart, "Computer-based experiments to measure $R C$," Phys. Teach. 38, 176-178 (March 2000).

5. Y. Kraftmakher, "Charge and energy stored in a capacitor," Phys. Teach. 50, 73-74 (Feb. 2012).

6. See, e.g., S. E. Rosenbaum, Basic Pharmacokinetics and Pharmacodynamics: An Integrated Textbook and Computer Simulations (Wiley, New Jersey, 2011).

Paul Janssen was professor (now retired) at the KU Leuven, working in the Semiconductor Physics Laboratory, where he was conducting research in the magnetic resonance field using medium to high magnetic fields in combination with far-infrared lasers. He is vividly interested in educational work and chaired physics courses in many science directions and pharmacy.

Mieke De Cock is an associate professor of physics in the KU Leuven, Belgium. She is responsible for the physics teaching training program and conducts research on the teaching and learning of physics. She also teaches the introductory physics courses to the first-year pharmacy students.

mieke.decock@fys.kuleuven.be 